\title{
Association of Leukocyte Telomere Length and Genes Involved in its Regulation With Oral Carcinoma
}

\author{
ZANETA JUMATOVAITE ${ }^{1}$, ALBERTAS KRIAUCIUNAS ${ }^{2}$, ALVITA VILKEVICIUTE $^{3}$, GRETA GEDVILAITE ${ }^{3}$, \\ VYKINTAS LIUTKEVICIUS ${ }^{4}$, VIRGILIJUS ULOZA ${ }^{4}$, ALINA SMALINSKIENE ${ }^{5}$ and RASA LIUTKEVICIENE ${ }^{3}$ \\ ${ }^{1}$ Medical Faculty, Lithuanian University of Health Sciences, Kaunas, Lithuania; \\ ${ }^{2}$ Department of Prosthodontics, Lithuanian University of Health Sciences, Kaunas, Lithuania; \\ ${ }^{3}$ Neuroscience Institute, Lithuanian University of Health Sciences, Kaunas, Lithuania; \\ ${ }^{4}$ Department of Otorhinolaryngology, Lithuanian University of Health Sciences, Kaunas, Lithuania; \\ ${ }^{5}$ Institute of Biological Systems and Genetics Research, \\ Lithuanian University of Health Sciences, Kaunas, Lithuania
}

\begin{abstract}
Background/Aim: This study aimed to determine the relationship between the relative leukocyte telomere length (RLTL) and gene polymorphisms involved in its regulation with the occurrence of oral squamous cell carcinoma (OSCC). Patients and Methods: Patients with OSCC and healthy subjects were examined. Genotyping and RLTL measurement were carried out using rPCR. Results: The OSCC group had longer telomeres than controls ( $p=0.001)$. Minor allele $T$ at TERF1rs1545827 may increase RLTL shortening $(p=0.047)$. TNKS2rs $10509639 \mathrm{~A} / G$ and $A / G+G / G$ genotypes were associated with a 2.6-fold increased odd $(p=0.012)$ and a 2.4-fold increased odd ( $p=0.019)$ of RLTL elongation compared to A/A genotype. The A/G genotype was associated with a 2.6-fold increased odd ( $p=0.011)$ compared to the $A / A+G / G$ genotypes. Each $G$ allele was associated with a 2.1-fold increased odd of longer RLTL ( $p=0.036)$. Conclusion: Longer telomeres were found in patients with OSCC than in controls. The TERF1 rs1545827 and the TNKS2 rs10509639 polymorphisms were associated with an increase in RLTL.
\end{abstract}

Oral squamous cell carcinoma (OSCC) accounts for $20 \%$ of all head and neck squamous cell carcinoma (HNSCC) cases worldwide, which makes it one of the most common types of malignant tumors of this anatomical region. This broad

This article is freely accessible online.

Correspondence to: Albertas Kriauciunas, Department of Prosthodontics, LUHS, Kaunas, Lithuania. Tel: +37 068968605 , e-mail albertas.kriauciunas@1smuni.lt

Key Words: Relative leukocyte telomere length, gene polymorphisms, oral squamous cell carcinoma. prevalence can be associated with risk factors, such as the use of tobacco products and alcohol consumption that are also associated with the development and progression of various chronic diseases. Early cancer diagnosis (stage I-II), rises the patient survival rate up to $70-90 \%$. For this purpose, new molecular biological markers could be of service in diagnosing the disease at its early stages (1). Molecular markers are indicators of normal biological, pathological and pharmacological molecular responses, which can provide useful information for diagnosis and prognosis (2).

Lately, the attention has been focused on telomeres and telomere length regulating genes, due to their importance in aging and the development of chronic diseases and malignant tumors. The name telomere itself originates from the Greek word "telos", which means the end, and "meros", which means a part (3). The main function of telomeres is to protect the ends of chromosomes from degradation and end-to-end fusions (4). There is also a telomere protein complex, called shelterin, that is incredibly important for the protection of the ends of chromosomes and the regulation of telomere length (5). Although telomeres in the human generative cells and embryotic stem cells are kept active by the help of telomerases, telomeres become shorter when somatic cells divide, because of insufficient telomerase expression (4). There is a significant number of genes and their coding proteins that are associated with the regulation of telomeres. TRF1 and TRF2 are negative telomere length regulators telomerase inhibitors, which means that overexpression of TRF1 and TRF2 causes shortening of telomeres $(6,7)$. Tankirase 2 (TNKS2) protects the linear chromosome structure (8). TERT gene encodes the catalytic subunit of telomerase and plays a major role in telomere DNA length preservation and carcinogenesis (9). Telomerase is a ribonucleic acid-protein complex, which is responsible for maintaining the length of telomeres (10). It is highly active 
Table I. Demographic characteristics.

\begin{tabular}{lccl}
\hline & Control group & OSCC & $p$ mean \\
\hline Age, Mean (SD) & $62.02(11.15)$ & $59.49(11.32)$ & $0.163^{*}$ \\
Gender, N, (\%) & $144(70.6)$ & $35(74.5)$ & $0.368^{* *}$ \\
$\quad$ Male & $60(29.4)$ & $12(25.5)$ & \\
Female & & & - \\
Stage, N (\%) & - & $2(4.3)$ & - \\
I & & $13(27.7)$ & \\
II & & $12(25.5)$ & \\
III & & $20(42.6)$ & \\
IV & & $1(2.2)$ & \\
G Status, N (\%) & & $36(78.3)$ & - \\
1 & & $9(19.6)$ & \\
2 & - &
\end{tabular}

OSCC: Oral squamous cell carcinoma; SD: standard deviation; *Student's $t$-test; **Fisher's Exact test; G status: tumor differentiation grade.

in cancer cells, resulting in unlimited replication potential and growth of the tumor (11).

It is particularly important to understand the mechanisms regulating telomere length and the relation between telomere length changes and various diseases. Discovery of new molecular markers is required to diagnose tumorous diseases, identify the risk groups for disease development, predict the response to treatment and carry out patients' observations in order to apply personalized treatment. For this reason, we assessed the associations of TERT rs2736098, TERTCLPTM1 rs401681, TRF1 rs1545827, rs10107605, TNKS2 rs10509639 and rs10509637, and TERF2 rs251796 gene polymorphisms and telomere length with OSCC.

\section{Materials and Methods}

All the procedures used in this study were approved by the Kaunas Regional Ethics Committee for Biomedical Research, Lithuania in compliance with ethical standards (permission number is BE-2-34). The study was conducted at the Department of Otorhinolaryngology, and Ophthalmology Laboratory of Neuroscience Institute, Lithuanian University of Health Sciences, Kaunas, Lithuania.

Study group. The current study included 47 patients (35 males and 12 females with a mean age of 59.5 years old) with a diagnosis of OSCC and 204 healthy control group subjects (144 males and 60 females, with a mean age of 62.02 years old) (Table I).

The patients included in the study had OSCC with no additional diseases, their general health status was good, and they had provided a written informed consent for participation in the study. The final diagnosis of OSCC was confirmed following histopathological investigation after a biopsy or surgery. Subjects chosen to participate as a control group did not have any oncological diseases, their general health status was good, and they provided a written informed consent for inclusion in the study.
Table II. Relationship between OSCC stage and relative leukocyte telomere length.

\begin{tabular}{lccccc}
\hline & \multicolumn{4}{c}{ OSCC stage } & $p$-Value* \\
\cline { 2 - 5 } & I & II & III & IV & \\
\hline $\begin{array}{l}\text { RLTL; median } \\
\text { (IQR) }\end{array}$ & $0.560(-)$ & $\begin{array}{c}1.719 \\
(5.273)\end{array}$ & $\begin{array}{c}1.505 \\
(3.424)\end{array}$ & $\begin{array}{c}1.952 \\
(3.573)\end{array}$ & $0.047 * *$ \\
\hline
\end{tabular}

IQR: Interquartile range; OSCC: oral squamous cell carcinoma; *MannWhitney $U$-test; $* *$ I stage $v s$. IV stage.

Table III. Relationship between OSCC G status (tumor differentiation grade) and relative telomere length.

\begin{tabular}{lcccc}
\hline & \multicolumn{2}{c}{ OSCC G status } & $p$-Value* \\
\cline { 2 - 4 } & 1 & 2 & 3 & \\
\hline $\begin{array}{l}\text { RLTL; median } \\
\text { (IQR) }\end{array}$ & - & $1.432(3.574)$ & $1.500(2.926)$ & $0.872 * *$ \\
\hline
\end{tabular}

IQR: Interquartile range; *Mann-Whitney $U$-test; **G2 vs. G3.

DNA extraction, genotyping, relative leukocyte telomere length measurement and statistical analysis. DNA extraction, genotyping and measurement of the relative leukocyte telomere length (RLTL) methods, as well as statistical analysis, have been described in detail in our previous studies $(12,13)$.

\section{Results}

OSCC patients had statistically significantly longer telomeres than healthy controls [median (IQR): 1.5 (3.34) vs. 0.95 (1.43)], $p=0.001$, respectively. Results are shown in Figure 1.

After analyzing patients' relative leukocyte telomere length in relation to the stage of the disease, we determined that OSCC patients with stage IV cancer had longer relative leukocyte telomere length than those with stage I [median (IQR): 1.952 (3.573) vs. $0.560(-), p=0.047$ ] (Table II). Analogous analysis was performed with different $\mathrm{G}$ status (tumor differentiation grade) groups of OSCC patients, but no statistically significant results were acquired (Table III).

We also determined statistically significant differences between telomere lengths of healthy control group individuals and OSCC patients with different stages of the disease (control group stage is marked with 0). OSCC patients with stage II and IV disease had longer relative leukocyte telomere length than controls $(p=0.014$ and $p=0.003$, respectively). Results are shown in Table IV.

TERT rs2736098, TERT-CLPTM1 rs401681, TRF1 rs1545827, rs10107605, TNKS2 rs10509639 and rs10509637, 


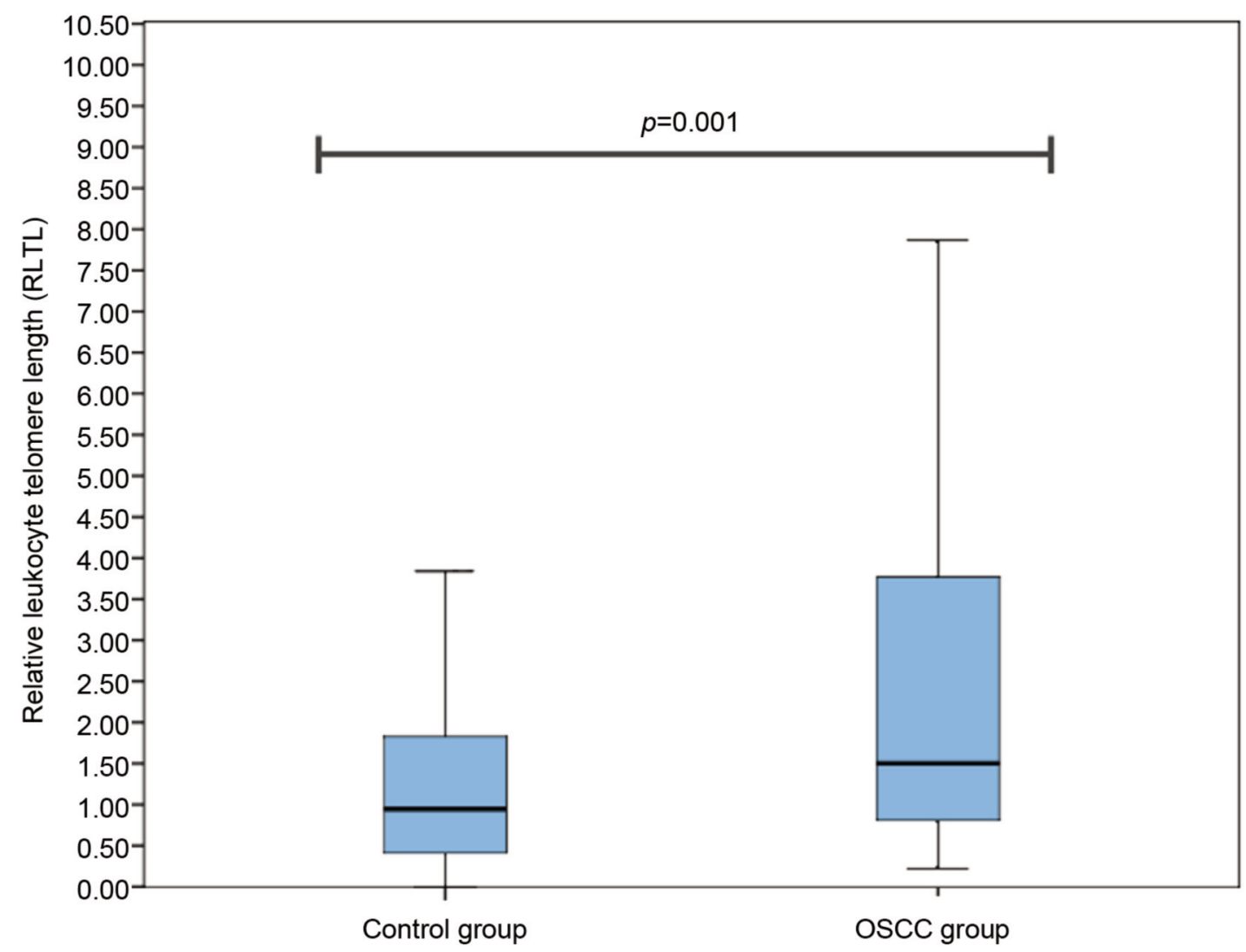

Figure 1. Relative leucocyte telomere length in the control and OSCC groups. Relative leukocyte telomere length (RLTL) in OSCC group versus control group are presented as box-and-whisker plots with the median and interquartile range. Mann-Whitney U-test was used to assess the differences of RLTL between OSCC and control groups.

TERF2 rs251796 matched the Hardy-Wainber equilibrium (HWE) ( $p>0.001)$ (Table V). After analyzing these SNPs' distribution, no statistically significant differences were found between the OSCC patients and control groups (Table VI).

Analysis of the SNPs (TERT rs2736098, TERT-CLPTM1 rs401681, TERF1 rs1545827, rs10107605, TNKS2 rs10509639 and rs10509637, TERF2 rs251796) and binary logistic regression association with the age and gender of patients with OSCC and healthy controls, did not reveal any statistically significant differences.

The relative leukocyte telomere length of the healthy control group individuals and OSCC patients was analyzed in relation to the SNPs (TERT rs2736098, TERT-CLPTM1 rs401681, TERF1 rs1545827, rs10107605, TNKS2 rs 10509639 and rs 10509637, TERF2 rs251796). This analysis showed no statistically significant results (Table VII). We also searched for an association between the control group subjects and OSCC patients' leukocyte telomere length and SNP genotypes. Analysis showed that at least one rare allele at TERF1, rs 1545827, increased the possibility of telomere shortening $(p=0.047)$ in the healthy control group. Analysis
Table IV. The comparison of relative leukocyte telomere length differences between four OSCC stages and the control group.

\begin{tabular}{ll}
\hline Stage & $p$-Value* \\
\hline 0 vs. I & 0.418 \\
0 vs. II & 0.014 \\
0 vs. III & 0.246 \\
0 vs. IV & 0.003
\end{tabular}

*Mann-Whitney $U$-test.

of the other polymorphisms showed no statistically significant results. Results are given in Table VIII and Table IX.

For further analysis, subjects were divided in two groups according to telomere length: short and long telomere groups. The TNKS2 rs10509639 genotype rate was found to be statistically significantly different between the short and long telomere groups $(p=0.022)$. Also, rs $10509639 \mathrm{G}$ allele was more common in subjects who had long telomeres $(p=0.036)$ (Table X). 
Table V. Hardy-Weinberg equilibrium analysis.

\begin{tabular}{|c|c|c|c|}
\hline SNP & Allele rate & Gene distribution & HWE $p$-Value \\
\hline \multirow[t]{2}{*}{ TERT rs2736098 } & C 0.73 & $17 / 77 / 110$ & 0.50 \\
\hline & Т 0.27 & & \\
\hline \multirow[t]{2}{*}{ TERT rs 401681} & C 0.62 & & \\
\hline & Т 0.38 & $26 / 105 / 73$ & 0.21 \\
\hline \multirow[t]{2}{*}{ TERF1 rs1545827 } & C 0.59 & & \\
\hline & Т 0.41 & $32 / 105 / 67$ & 0.39 \\
\hline \multirow[t]{2}{*}{ TERF1 rs10107605 } & A 0.87 & & \\
\hline & C 0.13 & $6 / 41 / 157$ & 0.11 \\
\hline \multirow[t]{2}{*}{ TNKS2 rs10509637 } & A 0.82 & & \\
\hline & G 0.18 & $8 / 59 / 137$ & 0.61 \\
\hline \multirow[t]{2}{*}{ TNKS2 rs10509639 } & А 0.92 & & \\
\hline & G 0.08 & $0 / 32 / 172$ & 0.22 \\
\hline \multirow[t]{2}{*}{ TERF2 rs251796 } & A 0.71 & & \\
\hline & G 0.29 & $22 / 76 / 106$ & 0.14 \\
\hline
\end{tabular}

SNP: Single-nucleotide polymorphism.

Binary logistic analysis was performed in order to evaluate the effect of TERT rs2736098, TERT-CLPTM1 rs401681, TERF1 rs1545827, rs10107605, TNKS2 rs10509639 and rs10509637, TERF2 rs251796 on the length of telomeres. Based on the analysis, the TNKS2 rs 10509639 polymorphism $\mathrm{A} / \mathrm{G}$ genotype and the $\mathrm{A} / \mathrm{G}$ and $\mathrm{G} / \mathrm{G}$ genotypes together increased the odd of telomere lengthening 2.0 -fold $(\mathrm{OR}=2.555 ; 95 \% \mathrm{CI}=1.227-5.318 ; p=0.012)$, and 2.4-fold $\quad(\mathrm{OR}=2.358 ; \quad 95 \% \mathrm{CI}=1.152-4.827 ; \quad p=0.019)$, respectively, in comparison to A/A genotype. Also, the odds ratio of telomere length was increased 2.6-fold by the $\mathrm{A} / \mathrm{G}$ genotype, in comparison to $\mathrm{A} / \mathrm{A}$ and $\mathrm{G} / \mathrm{G}$ genotypes together $(\mathrm{OR}=2.578 ; 95 \% \mathrm{CI}=1.239-5.366 ; p=0.011)$ and each $\mathrm{G}$ allele increased the odd 2.1-fold $(\mathrm{OR}=2.090 ; 95 \% \mathrm{CI}=1.050-4.160$; $p=0.036$ ) (Table XI).

\section{Discussion}

Despite the development of modern medical technologies and the increasing number of diagnostic methods, the survival rate of patients with advanced (III-IV stage) HNSCC, as well as with OSCC, has increased only up to $65 \%$ (14). In order to increase the survival rate of patients, research is focused on identifying new markers such as telomere length and gene polymorphisms regulating telomere length (TERT rs2736098, TERT-CLPTM1 rs401681, TRF1 rs1545827, rs10107605, TNKS2 rs10509639 and rs10509637, TERF2 rs251796), which could be useful for diagnosis and treatment.

Results from analyses of telomere length in cancer patients are scarce and inconsistent. Our results revealed that OSCC patients had statistically significantly longer telomeres than healthy controls [median (IQR): 1.5 (3.34) vs. 0.95 (1.43),
Table VI. TERT rs2736098, TERT-CLPTM1 rs401681, TRF1 rs1545827, rs10107605, TNKS2 rs10509639 and rs10509637, TERF2 rs251796 genotype polymorphisms and alleles' frequencies in OSCC patients and control group individuals.

\begin{tabular}{|c|c|c|c|c|}
\hline SNP & Genotype & $\begin{array}{c}\text { Control group } \\
\mathrm{N}(\%)\end{array}$ & $\begin{array}{l}\text { Floor of the } \\
\text { mouth cancer } \\
\mathrm{N}(\%)\end{array}$ & $p$-Value* \\
\hline$T E R T$ & $\mathrm{CC}$ & $110(53.9)$ & $24(51.1)$ & 0.936 \\
\hline \multirow[t]{5}{*}{ rs 2736098} & $\mathrm{CT}$ & $77(37.7)$ & $19(40.4)$ & \\
\hline & TT & $17(8.3)$ & $4(8.5)$ & \\
\hline & Alleles & 0.766 & & \\
\hline & C & $297(72.8)$ & 67 (71.3) & \\
\hline & $\mathrm{T}$ & $111(27.2)$ & $27(28.7)$ & \\
\hline \multirow{6}{*}{$\begin{array}{l}\text { IERI-CLPIMI } \\
\text { rs401681 }\end{array}$} & $\mathrm{CC}$ & $73(35.8)$ & 19 (40.4) & 0.453 \\
\hline & $\mathrm{CT}$ & $105(51.5)$ & $25(53.2)$ & \\
\hline & TT & $26(12.7)$ & $3(6.4)$ & \\
\hline & Alleles & 0.320 & & \\
\hline & C & $251(61.5)$ & $63(67.0)$ & \\
\hline & $\mathrm{T}$ & $157(38.5)$ & $31(33)$ & \\
\hline TERF 1 & $\mathrm{CC}$ & $67(32.8)$ & $11(23.4)$ & 0.447 \\
\hline \multirow[t]{5}{*}{ rs1545827 } & $\mathrm{CT}$ & $105(51.5)$ & $28(59.6)$ & \\
\hline & TT & $32(15.7)$ & $8(17.0)$ & \\
\hline & Alleles & & & \\
\hline & C & 239 (58.6) & $50(53.2)$ & 0.341 \\
\hline & $\mathrm{T}$ & 169 (41.4) & $44(46.8)$ & \\
\hline TERF1 & AA & $157(77.0)$ & $38(80.9)$ & 0.479 \\
\hline \multirow[t]{5}{*}{ rs 10107605} & $\mathrm{AC}$ & $41(20.1)$ & $9(19.1)$ & \\
\hline & $\mathrm{CC}$ & $6(2.9)$ & $0(0.0)$ & \\
\hline & Alleles & & & \\
\hline & A & $355(87.0)$ & $85(90.4)$ & 0.364 \\
\hline & $\mathrm{C}$ & $53(13.0)$ & $9(9.6)$ & \\
\hline TNKS2 & AA & $137(67.2)$ & $34(72.3)$ & 0.749 \\
\hline \multirow[t]{5}{*}{ rs10509637 } & $\mathrm{AG}$ & $59(28.9)$ & $11(23.4)$ & \\
\hline & GG & $8(3.9)$ & $2(4.3)$ & \\
\hline & Alleles & & & \\
\hline & A & 333 (81.6) & $79(84.0)$ & 0.581 \\
\hline & $\mathrm{G}$ & $75(18.4)$ & $15(16.0)$ & \\
\hline TNKS2 & AA & $172(84.3)$ & $36(76.6)$ & 0.070 \\
\hline \multirow[t]{5}{*}{ rs 10509639} & AG & $32(15.7)$ & $10(21.3)$ & \\
\hline & GG & $0(0.0)$ & $1(2.1)$ & \\
\hline & Alleles & & & \\
\hline & A & $376(92.2)$ & $82(87.2)$ & 0.128 \\
\hline & $\mathrm{G}$ & $32(7.8)$ & $12(12.8)$ & \\
\hline$T E R F 2$ & AA & $106(52.0)$ & $22(46.8)$ & 0.805 \\
\hline \multirow[t]{5}{*}{ rs251796 } & AG & $76(37.3)$ & $19(40.4)$ & \\
\hline & GG & $22(10.8)$ & $6(12.8)$ & \\
\hline & Alleles & & & \\
\hline & A & $288(70.6)$ & $63(67.0)$ & 0.497 \\
\hline & G & $120(29.4)$ & $31(33.0)$ & \\
\hline
\end{tabular}

SNP: Single-nucleotide polymorphism; *Pearson's $\chi^{2}$ test.

$p=0.001$, respectively]. A number of studies have examined associations between telomere length and cancer, but only few studies have focused on head and neck cancer, telomere length and telomere length regulating gene polymorphisms. Zhang et al. have demonstrated that short telomere length in peripheral blood mononuclear cells (PBMCs) was strongly associated 
Jumatovaite et al: Leukocyte Telomere and Oral Carcinoma

Table VII. Association between relative leukocyte telomere length and SNP genotypes.

\begin{tabular}{|c|c|c|c|c|c|c|}
\hline SNP & $\begin{array}{c}22 \\
\text { Median (IQR) }\end{array}$ & $\begin{array}{c}11+12 \\
\text { Median (IQR) }\end{array}$ & $p$-Value* & $\begin{array}{c}12+22 \\
\text { Median (IQR) }\end{array}$ & $\begin{array}{c}11 \\
\text { Median (IQR) }\end{array}$ & $p$-Value* \\
\hline TERT rs 2736098 & $1.176(2.05)$ & $0.997(1.51)$ & 0.313 & $1.11(1.85)$ & $0.964(1.27)$ & 0.297 \\
\hline TERT-CLPTM1 rs401681 & $0.883(1.19)$ & $1.012(1.82)$ & 0.224 & $1.052(1.54)$ & $0.881(1.68)$ & 0.294 \\
\hline TRF1 rs1545827 & $1.007(1.25)$ & $0.997(1.63)$ & 0.6 & $0.933(1.44)$ & $1.057(1.82)$ & 0.061 \\
\hline TRF1 rs10107605 & $0.745(1.58)$ & $1.017(1.59)$ & 0.258 & $0.965(1.13)$ & $1.007(1.81)$ & 0.497 \\
\hline TNKS2 rs 10509637 & $1.298(1.52)$ & $0.99(1.57)$ & 0.458 & $1.010(2.04)$ & $1.003(1.39)$ & 0.557 \\
\hline TNKS2 rs 10509639 & - & 1.005 (1.6) & - & $1.275(1.56)$ & $0.954(1.61)$ & 0.187 \\
\hline TERF2 rs 251796 & $0.857(1.81)$ & $1.028(1.6)$ & 0.668 & $0.94(1.61)$ & $1.112(1.59)$ & 0.588 \\
\hline
\end{tabular}

11-homozygotes, who have the more frequent allele, 12-heterozygotes, 22-homozygotes, who have the rarer allele; SNP: single-nucleotide polymorphism; *Mann-Whitney $U$-test.

Table VIII. Association between healthy control group individuals' relative leukocyte telomere length and SNP genotypes.

\begin{tabular}{|c|c|c|c|c|c|c|}
\hline SNP & $\begin{array}{c}22 \\
\text { Median (IQR) }\end{array}$ & $\begin{array}{c}11+12 \\
\text { Median (IQR) }\end{array}$ & $p$-Value* & $\begin{array}{c}12+22 \\
\text { Median (IQR) }\end{array}$ & $\begin{array}{c}11 \\
\text { Median (IQR) }\end{array}$ & $p$-Value* \\
\hline TERT rs2736098 & $0.878(1.9)$ & $0.959(1.39)$ & 0.709 & $0.967(1.84)$ & $0.933(1.2)$ & 0.453 \\
\hline TERT-CLPTM1 rs 401681 & $0.937(1.16)$ & $0.947(1.62)$ & 0.596 & $1.003(1.37)$ & $0.858(1.72)$ & 0.478 \\
\hline TRF1 rs1545827 & $0.832(1.43)$ & $0.957(1.5)$ & 0.552 & $0.825(1.29)$ & $1.043(1.75)$ & 0.047 \\
\hline TRF1 rs10107605 & $0.745(1.58)$ & $0.957(1.44)$ & 0.347 & $0.954(1.19)$ & $0.94(1.57)$ & 0.781 \\
\hline TNKS2 rs 10509637 & $1.175(1.51)$ & $0.933(1.43)$ & 0.84 & $0.883(1.66)$ & $0.959(1.33)$ & 0.818 \\
\hline TNKS2 rs10509639 & - & $0.947(1.43)$ & - & $1.233(1.58)$ & $0.884(1.34)$ & 0.246 \\
\hline TERF2 rs 251796 & $0.731(1.61)$ & $0.963(1.42)$ & 0.75 & $0.88(1.41)$ & $1.005(1.54)$ & 0.714 \\
\hline
\end{tabular}

11-homozygotes, who have the more frequent allele, 12-heterozygotes, 22-homozygotes, who have the rarer allele; SNP: single nucleotide polymorphism; *Mann-Whitney $U$-test.

Table IX. Association between relative leukocyte telomere length and SNP genotypes in OSCC patients.

\begin{tabular}{|c|c|c|c|c|c|c|}
\hline SNP & $\begin{array}{c}22 \\
\text { Median (IQR) }\end{array}$ & $\begin{array}{c}11+12 \\
\text { Median (IQR) }\end{array}$ & $p$-Value* & $\begin{array}{c}12+22 \\
\text { Median (IQR) }\end{array}$ & $\begin{array}{c}11 \\
\text { Median (IQR) }\end{array}$ & $p$-Value* \\
\hline TERT rs2736098 & $4.172(5.1)$ & $1.479(3)$ & 0.159 & $1.952(4.09)$ & $1.432(2.07)$ & 0.448 \\
\hline TERT-CLPTM1 rs401681 & $0.693(-)$ & $1.562(3.76)$ & 0.147 & $1.782(4.9)$ & $1.21(1.67)$ & 0.095 \\
\hline TRF1 rs 1545827 & $1.21(4.58)$ & $1.782(3.21)$ & 0.773 & $1.5(3.78)$ & $2.014(3.09)$ & 0.56 \\
\hline TRF1 rs 10107605 & - & $1.5(3.34)$ & - & $1.21(2.01)$ & $1.782(4.26)$ & 0.385 \\
\hline TNKS2 rs10509637 & $4.862(-)$ & $1.479(3.02)$ & 0.22 & $3.531(4.2)$ & $1.277(2.17)$ & 0.147 \\
\hline TNKS2 rs10509639 & - & $1.562(3.53)$ & - & $1.624(2.42)$ & $1.5(3.96)$ & 0.91 \\
\hline TERF2 rs 251796 & $1.103(4.27)$ & $1.625(3.67)$ & 0.577 & $1.31(4.12)$ & $1.562(2.24)$ & 0.715 \\
\hline
\end{tabular}

11-homozygotes, who have the more frequent allele, 12-heterozygotes, 22-homozygotes, who have the rarer allele; SNP: single-nucleotide polymorphism; *Mann-Whitney $U$-test.

with a moderately increased risk of oropharyngeal squamous cell carcinoma; however, not with an increased risk of oral cavity cancer. Associations between telomere length and a higher risk of HPV16-positive oropharyngeal carcinoma and tumor HPV16 status have also been revealed (15). Bau et al. have observed that short leukocyte telomere length was associated with an increased risk of developing oral premalignant lesions and precursors of OSCC (16). A study performed by Alves-Paiva et al. has also found that RLTL was significantly shorter in patients with head and neck cancer (HNC) in comparison to healthy controls $(p=0.0003)$. Patients with shortest RLTL had an increased risk of developing HNC $(p<0.0001)$. No significant correlation was observed between RLTL and patients' clinical features and personal habits (17). 
Table X. Relative telomere length and SNP association.

\begin{tabular}{|c|c|c|c|c|}
\hline SNP & Genotype & $\begin{array}{c}\text { Short } \\
\text { telomeres } \\
\mathrm{N}(\%)\end{array}$ & $\begin{array}{l}\text { Long } \\
\text { telomeres } \\
\mathrm{N}(\%)\end{array}$ & $p$-Value* \\
\hline$T E R T$ & $\mathrm{CC}$ & $68(55.7)$ & $64(52.0)$ & 0.844 \\
\hline \multirow{5}{*}{ rs2736098 } & $\mathrm{CT}$ & $44(36.1)$ & $48(39.0)$ & \\
\hline & $\mathrm{TT}$ & $10(8.2)$ & $11(8.9)$ & \\
\hline & Alleles & & & \\
\hline & $\mathrm{C}$ & $180(73.8)$ & $176(71.5)$ & 0.580 \\
\hline & $\mathrm{T}$ & $64(26.2)$ & $70(28.5)$ & \\
\hline TERT-CLPTMI & $\mathrm{CC}$ & $50(41.0)$ & $40(32.5)$ & 0.226 \\
\hline \multirow[t]{5}{*}{ rs 401681} & $\mathrm{CT}$ & 56 (45.9) & $70(56.9)$ & \\
\hline & $\mathrm{TT}$ & $16(13.1)$ & $13(10.6)$ & \\
\hline & Alleles & & & \\
\hline & $\mathrm{C}$ & $156(63.9)$ & $150(61.0)$ & 0.499 \\
\hline & $\mathrm{T}$ & $88(36.1)$ & $96(39.0)$ & \\
\hline TERF1 & $\mathrm{CC}$ & $33(27.0)$ & $44(35.8)$ & 0.282 \\
\hline \multirow[t]{5}{*}{ rs 1545827} & $\mathrm{CT}$ & $70(57.4)$ & $59(48.0)$ & \\
\hline & $\mathrm{TT}$ & 19 (15.6) & $20(16.3)$ & \\
\hline & Alleles & & & \\
\hline & $\mathrm{C}$ & $136(55.7)$ & $147(59.8)$ & 0.368 \\
\hline & $\mathrm{T}$ & $108(44.3)$ & $99(40.2)$ & \\
\hline TERF1 & AA & $94(77.0)$ & 97 (78.9) & 0.247 \\
\hline \multirow[t]{5}{*}{ rs 10107605} & $\mathrm{AC}$ & $23(18.9)$ & $25(20.3)$ & \\
\hline & $\mathrm{CC}$ & $5(4.1)$ & $1(0.8)$ & \\
\hline & Alleles & & & \\
\hline & A & $211(86.5)$ & $219(89.0)$ & 0.389 \\
\hline & $\mathrm{C}$ & $33(13.5)$ & $27(11.0)$ & \\
\hline TNKS2 & AA & $83(68.0)$ & $84(68.3)$ & 0.399 \\
\hline \multirow[t]{5}{*}{ rs10509637 } & $\mathrm{AG}$ & $36(29.5)$ & $32(26.0)$ & \\
\hline & GG & $3(2.5)$ & $7(5.7)$ & \\
\hline & Alleles & & & \\
\hline & A & $202(82.8)$ & $200(81.3)$ & 0.668 \\
\hline & $\mathrm{G}$ & $42(17.2)$ & 46 (18.7) & \\
\hline TNKS2 & $\mathrm{AA}$ & $109(89.3)$ & $96(78.0)$ & 0.022 \\
\hline \multirow[t]{5}{*}{ rs 10509639} & $\mathrm{AG}$ & $12(9.8)$ & $27(22.0)$ & \\
\hline & GG & $1(0.8)$ & $0(0)$ & \\
\hline & Alleles & & & \\
\hline & A & $230(94.3)$ & $219(89.0)$ & 0.036 \\
\hline & $\mathrm{G}$ & $14(5.7)$ & $27(11.0)$ & \\
\hline TERF2 & $\mathrm{AA}$ & $56(45.9)$ & $70(56.9)$ & 0.185 \\
\hline \multirow[t]{5}{*}{ rs 251796} & $\mathrm{AG}$ & $49(40.2)$ & $42(34.1)$ & \\
\hline & GG & $17(13.9)$ & $11(8.9)$ & \\
\hline & Alleles & & & \\
\hline & A & $161(66.0)$ & $182(74.0)$ & 0.053 \\
\hline & G & $83(34.0)$ & $64(26.0)$ & \\
\hline
\end{tabular}

SNP: Single nucleotide polymorphism.

The opposite results were revealed by Liu et al. who have reported that relative telomere length may be not important in HNSCC carcinogenesis (18). Oh et al., have suggested that the advancement of tumors may correlate with long telomere length and a poor prognosis (19). The authors stated that increased telomere length indicates that telomere maintenance may also be important for the progression of
Table XI. TERT rs2736098, TERT-CLPTM1 rs401681, TRF1 rs1545827, rs10107605, TNKS2 rs10509639 and rs10509637, TERF2 rs251796 binary logistic regression analysis.

\begin{tabular}{|c|c|c|c|c|}
\hline Model & $\begin{array}{l}\text { Genotype/ } \\
\text { Allele }\end{array}$ & OR $(95 \% \mathrm{CI})$ & $p$-Value & AIC \\
\hline \multicolumn{5}{|l|}{ TERT rs 2736098} \\
\hline \multirow[t]{2}{*}{ Co-dominant } & $\mathrm{C} / \mathrm{T}$ & $1.159(0.680-1.975)$ & 0.587 & 343.299 \\
\hline & $\mathrm{T} / \mathrm{T}$ & $1169(0.465-2.938)$ & 0.740 & \\
\hline Dominant & $\mathrm{C} / \mathrm{T}+\mathrm{T} / \mathrm{T}$ & $1161(0.702-1.919)$ & 0.561 & 341.300 \\
\hline Recessive & $\mathrm{T} / \mathrm{T}$ & $1.100(0.449-2.693)$ & 0.835 & 341.594 \\
\hline Over-dominant & $\mathrm{C} / \mathrm{T}$ & $1.135(0.676-1.904)$ & 0.633 & 341.409 \\
\hline Additive & $\mathrm{T}$ & $1.112(0.755-1.638)$ & 0.591 & 341.348 \\
\hline \multicolumn{5}{|l|}{ TERT rs 401681} \\
\hline \multirow[t]{2}{*}{ Co-dominant } & $\mathrm{C} / \mathrm{T}$ & $1.562(0.907-2.693)$ & 0.108 & 340.659 \\
\hline & $\mathrm{T} / \mathrm{T}$ & $1.016(0.438-2.357)$ & 0.971 & \\
\hline Dominant & $\mathrm{C} / \mathrm{T}+\mathrm{T} / \mathrm{T}$ & $1.441(0.855-2.429)$ & 0.170 & 339.747 \\
\hline Recessive & $\mathrm{T} / \mathrm{T}$ & $0.783(0.359-1.706)$ & 0.538 & 341.257 \\
\hline Over-dominant & $\mathrm{C} / \mathrm{T}$ & $1.557(0.940-2.577)$ & 0.085 & 338.660 \\
\hline Additive & $\mathrm{T}$ & $1.150(0.782-1.691)$ & 0.477 & 341.131 \\
\hline \multicolumn{5}{|c|}{ TERF1 rs1545827 } \\
\hline \multirow[t]{2}{*}{ Co-dominant } & $\mathrm{C} / \mathrm{T}$ & $0.632(0.358-1.117)$ & 0.114 & 341.101 \\
\hline & $\mathrm{T} / \mathrm{T}$ & $0.789(0.364-1.711)$ & 0.549 & \\
\hline Dominant & $\mathrm{C} / \mathrm{T}+\mathrm{T} / \mathrm{T}$ & $0.666(0.387-1.147)$ & 0.142 & 339.470 \\
\hline Recessive & $\mathrm{T} / \mathrm{T}$ & $1.053(0.531-2.088)$ & 0.883 & 341.616 \\
\hline Over-dominant & $\mathrm{C} / \mathrm{T}$ & $0.685(0.414-1.133)$ & 0.141 & 339.459 \\
\hline Additive & $\mathrm{T}$ & $0.836(0.574-1.216)$ & 0.349 & 340.757 \\
\hline \multicolumn{5}{|c|}{ TERF 1 rs 10107605} \\
\hline \multirow[t]{2}{*}{ Co-dominant } & $\mathrm{A} / \mathrm{C}$ & $1.053(0.559-1.984)$ & 0.872 & 340.601 \\
\hline & $\mathrm{C} / \mathrm{C}$ & $0.194(0.022-1.690)$ & 0.138 & \\
\hline Dominant & $\mathrm{A} / \mathrm{C}+\mathrm{C} / \mathrm{C}$ & $0.900(0.492-1.647)$ & 0.732 & 341.521 \\
\hline Recessive & $\mathrm{C} / \mathrm{C}$ & $0.192(0.022-1.666)$ & 0.134 & 338.626 \\
\hline Over-dominant & $\mathrm{A} / \mathrm{C}$ & $1.098(0.584-2.065)$ & 0.772 & 341.554 \\
\hline Additive & $\mathrm{C}$ & $0.803(0.477-1.353)$ & 0.411 & 340.956 \\
\hline \multicolumn{5}{|c|}{ TNKS2 rs 10509637} \\
\hline \multirow[t]{2}{*}{ Co-dominant } & $\mathrm{A} / \mathrm{G}$ & $0.878(0.499-1.545)$ & 0.652 & 341.755 \\
\hline & $\mathrm{G} / \mathrm{G}$ & $2.306(0.576-9.220)$ & 0.238 & \\
\hline Dominant & $\mathrm{A} / \mathrm{G}+\mathrm{G} / \mathrm{G}$ & $0.988(0.577-1.692)$ & 0.965 & 341.636 \\
\hline Recessive & $\mathrm{G} / \mathrm{G}$ & $2.394(0.604-9.481)$ & 0.214 & 339.958 \\
\hline Over-dominant & $\mathrm{A} / \mathrm{G}$ & $0.840(0.480-1.471)$ & 0.542 & 341.265 \\
\hline Additive & $\mathrm{G}$ & $1.100(0.702-1.724)$ & 0.677 & 341.464 \\
\hline \multicolumn{5}{|c|}{ TNKS2 rs 10509639} \\
\hline \multirow[t]{2}{*}{ Co-dominant } & $\mathrm{A} / \mathrm{G}$ & $2.555(1.227-5.318)$ & 0.012 & 335.510 \\
\hline & $\mathrm{G} / \mathrm{G}$ & - & 1.000 & \\
\hline Dominant & $\mathrm{A} / \mathrm{G}+\mathrm{G} / \mathrm{G}$ & $2.358(1.152-4.827)$ & 0.019 & 335.812 \\
\hline Recessive & $\mathrm{G} / \mathrm{G}$ & - & 1.000 & 340.239 \\
\hline Over-dominant & $\mathrm{A} / \mathrm{G}$ & $2.578(1.239-5.366)$ & 0.011 & 335.769 \\
\hline Additive & $\mathrm{G}$ & $2.090(1.050-4.160)$ & 0.036 & 336.996 \\
\hline \multicolumn{5}{|l|}{ TERF2 rs 251796} \\
\hline \multirow[t]{2}{*}{ Co-dominant } & $\mathrm{A} / \mathrm{G}$ & $0.686(0.399-1.179)$ & 0.172 & 340.249 \\
\hline & $\mathrm{G} / \mathrm{G}$ & $0.518(0.224-1.194)$ & 0.123 & \\
\hline Dominant & $\mathrm{A} / \mathrm{G}+\mathrm{G} / \mathrm{G}$ & $0.642(0.388-1.064)$ & 0.085 & 338.660 \\
\hline Recessive & $\mathrm{G} / \mathrm{G}$ & $0.607(0.272-1.355)$ & 0.223 & 340.121 \\
\hline Over-dominant & $\mathrm{A} / \mathrm{G}$ & $0.772(0.459-1.299)$ & 0.330 & 340.687 \\
\hline Additive & G & $0.708(0.488-1.027)$ & 0.069 & 338.274 \\
\hline
\end{tabular}

OR: Odds ratio; CI: confidence interval; AIC: Akaike information criterion. 
HNSCC (20). Our results are inconsistent with previous studies, except for the study by $\mathrm{Oh}$ et al. Also, longer telomeres can increase the possibility of cancer development by stimulating cell immortality, and causing genome instability; the association between long telomeres and an advanced stage with lower survival rate has been found in colorectal and liver carcinoma patients $(19,21,22)$.

Therefore, there are many studies suggesting that RLTL is associated with other types of cancer, and that both short and long telomeres can be associated with cancer development (23-27). However, a big meta-analysis performed by Weischer et al. has shown that RLTL is not associated with cancer risk (25). Da-Tian Bau et al. have stated that different cancers have different etiology, and pooling all cancer types together may mask the significant associations of RLTL with individual cancer types (16).

In our study, the TERF1 rs 1545827 polymorphism was associated with an increased RLTL while the TNKS2 rs 10509639 polymorphism was associated with longer RLTL. Ying Bao et al. have found that the TERT rs401681 rarer allele (A) was statistically significantly associated with telomere shortening $(p=0.023)$ in pancreatic cancer patients (28). Carriers of the $\mathrm{C}$ allele of the rs 2736100 polymorphism were statistically significantly associated with longer telomeres and a higher risk of cancer, while the A allele carriers were associated with shorter telomeres (29). TERT rs2736098 polymorphism increases the risk of lung, heart, bladder cancer and other malignancies (30). Also, Liu et al. have found that TERT rs2736098 with TERT-CLPTM1 rs401681 genotype variants are statistically significantly associated with reduced chances of having HNSCC ( $p=0.048)$ (31). TERF2 rs251796 G variant increases the risk for lung cancer $(p=0.03$ ) (32). The TNKS2 rs 10509637 rare allele $\mathrm{G}$ was associated with an increased risk of breast cancer ( $p=0.006$ ) (33). Furthermore, several studies have examined the association of single nucleotide polymorphisms with telomere regulation. For example, in a metanalysis performed in 2013, Bojesen et al. have found that the TERT gene rs7705526 rarer allele showed statistically significant association with longer telomeres $\left(p=2.3 \times 10^{-14}\right)$ and increases the possibility of low malignancy ovarian cancer $\left(p=1.3 \times 10^{-15}\right)(34)$. The results of other metanalyses showed that the TERT gene rs7726159 rarer allele (A), showed statistically significant associations with glioma and lung cancer $\left(p=4.38 \times 10^{-36}\right)$, but negative associations with testicular cancer, prostate cancer and pancreatic cancer $\left(p=5.07 \times 10^{-6}\right)(35)$. Research on TNKS2 rs1340420 showed that the $\mathrm{G} / \mathrm{G}$ and $\mathrm{A} / \mathrm{G}$ genotypes reduced the risk of lung adenocarcinoma $(p=0.03)$; whereas the TNKS2 rs 1770474 rarer $(\mathrm{T})$ allele increased the risk of lung squamous cell carcinoma in women 4.7 times $(p=0.049)$ (36).

In order to evaluate TERT rs2736098, TERT-CLPTM1 rs401681, TRF1 rs1545827, rs10107605, TNKS2 rs10509639 and rs10509637, TERF2 rs251796 polymorphisms' association with OSCC and telomere length changes, more subjects have to be analysed.

\section{Conclusion}

The relative telomere length was significantly higher in the group of OSCC patients compared to healthy controls. In OSCC patients, the TERF1 rs 1545827 and TNKS2 rs 10509639 polymorphisms were associated with increased relative telomere length.

\section{Conflicts of Interest}

None of the Authors have any conflicts of interest related to this submission.

\section{Authors' Contributions}

R.L., A.V., G.G., A.K., A.S., and V.U. designed research; Z.J., R.L., A.V., G.G., A.K., and V.U. performed research; A.V., G.G., Z.J., and V.U. analyzed data; and R.L., A.V., G.G., A.K., V.L., A.S., and V.U., wrote the paper.

\section{Acknowledgements}

The Authors would like to thank Thermo Fisher Scientific (Lithuania, Vilnius) for providing reagents for this study. This project has received funding from European Social Fund (project No 09.3.3-LMT-K-712-15-0210) under grant agreement with the Research Council of Lithuania (LMTLT).

\section{References}

1 Jafari A, Najafi Sh, Moradi F, Kharazifard M and Khami M: Delay in the diagnosis and treatment of oral cancer. J Dent (Shiraz) 14(3): 146-150, 2013. PMID: 24724136.

2 Khurshid Z, Zafar MS, Khan RS, Najeeb S, Slowey PD and Rehman IU: Role of salivary biomarkers in oral cancer detection. Adv Clin Chem 86: 23-70, 2018. PMID: 30144841. DOI: $10.1016 /$ bs.acc 2018.05 .002

3 Danese E and Lippi G: Telomere length: is the future in our "ends"? Ann Transl Med 6(13): 280-280, 2018. PMID: 30094266. DOI: 10.21037/atm.2018.06.24

4 Muraki K, Nyhan K, Han L and Murnane JP: Mechanisms of telomere loss and their consequences for chromosome instability. Front Oncol 2, 2012. PMID: 23061048. DOI: $10.3389 /$ fonc. 2012.00135

5 O'Sullivan RJ and Karlseder J: Telomeres: protecting chromosomes against genome instability. Nat Rev Mol Cell Biol 11(3): 171-181, 2010. PMID: 20125188. DOI: 10.1038/nrm2848

6 Court R, Chapman L, Fairall L and Rhodes D: How the human telomeric proteins TRF1 and TRF2 recognize telomeric DNA: a view from high-resolution crystal structures. EMBO Rep 6(1): 39-45, 2005. PMID: 15608617. DOI: 10.1038/sj.embor.7400314

7 Bellon M, Datta A, Brown M, Pouliquen JF, Couppie P, Kazanji $\mathrm{M}$ and Nicot $\mathrm{C}$ : Increased expression of telomere length 
regulating factors TRF1, TRF2 and TIN2 in patients with adult T-cell leukemia. Int J Cancer 119(9): 2090-2097, 2006. PMID: 16786598. DOI: $10.1002 / \mathrm{ijc} .22026$

8 Kang H-W, Wang F, Wei Q, Zhao YF, Liu M, Li X and Tang H: miR-20a promotes migration and invasion by regulating TNKS2 in human cervical cancer cells. FEBS Lett 586(6): 897-904, 2012. PMID: 22449978. DOI: 10.1016/j.febslet.2012.02.020

9 Zhou M, Jiang B, Xiong M and Zhu X: Association between TERT rs2736098 polymorphisms and cancer Risk-A metaanalysis. Front Physiol 9, 2018. PMID: 29695979. DOI: 10.3389/fphys.2018.00377

10 Hornsby PJ: Telomerase and the aging process. Exper Gerontol 42(7): 575-581, 2007. PMID: 17482404. DOI: 10.1016/j.exger. 2007.03.007

11 Lee YH, Chen YY, Yeh YL, Wang YJ and Chen RJ: Stilbene compounds inhibit tumor growth by the induction of cellular senescence and the inhibition of telomerase activity. Int J Mol Sci 20(11): 2716, 2019. PMID: 31159515. DOI: 10.3390/ijms20112716

12 Liutkeviciene R, Vilkeviciute A, Streleckiene G, Kriauciuniene L, Chaleckis R and Deltuva VP: Associations of cholesteryl ester transfer protein (CETP) gene variants with predisposition to agerelated macular degeneration. Gene 636: 30-35, 2017. PMID: 28918250. DOI: $10.1016 /$ j.gene.2017.09.022

13 Gedvilaite G, Vilkeviciute A, Kriauciuniene L, Banevičius M and Liutkeviciene R: The relationship between leukocyte telomere length and TERT, TRF1 single nucleotide polymorphisms in healthy people of different age groups. Biogerontol 21(1): 57-67, 2020. PMID: 31646401. DOI: 10.1007/s10522-019-09843-0

14 Koole K, Clausen MJAM, Es RJJV, Kempen PMW, Melchers LJ, Koole R, Langendijk JA, Diest PJ, Roodenburg JLN and Willems SM: FGFR Family members protein expression as prognostic markers in oral cavity and oropharyngeal squamous cell carcinoma. Mol Diagn Ther 20(4): 363-374, 2016. PMID: 27278367. DOI: $10.1007 / \mathrm{s} 40291-016-0204-5$

15 Zhang Y, Sturgis EM, Dahlstrom KR, Wen J, Liu H, Wei Q, Li $\mathrm{G}$ and Liu Z: Telomere length in peripheral blood lymphocytes contributes to the development of HPV-associated oropharyngeal carcinoma. Cancer Res 73(19): 5996-6003, 2013. PMID: 23928994. DOI: 10.1158/0008-5472.can-13-0881

16 Bau DT, Lippman SM, Xu E, Gong Y, Lee JJ, Wu X and Gu J: Short telomere lengths in peripheral blood leukocytes are associated with an increased risk of oral premalignant lesion and oral squamous cell carcinoma. Cancer 119(24): 4277-4283, 2013. PMID: 24105340. DOI: $10.1002 /$ cncr. 28367

17 Alves-Paiva RM, Gutierrez-Rodrigues F, Pereira-Martins DA, Figueiredo DLA, Cle DV, Conti-Freitas LC, Mamede RCM and Calado RT: Short telomere length in peripheral blood leukocytes in head and neck cancer: Findings in a Brazilian cohort. Head Neck 41(3): 672-677, 2018. PMID: 30589155. DOI: 10.1002/hed.25472

18 Liu Z, Ma H, Wei S, Li G, Sturgis EM and Wei Q: Telomere length and TERT functional polymorphisms are not associated with risk of squamous cell carcinoma of the head and neck. Cancer Epidemiol Biomarkers Prev 20(12): 2642-2645, 2011. PMID: 21994403. DOI: 10.1158/1055-9965 .epi-11-0890

19 Oh B-K, Kim H, Park YN, Yoo JE, Choi J, Kim KS, Lee JJ and Park C: High telomerase activity and long telomeres in advanced hepatocellular carcinomas with poor prognosis. Laboratory Invest 88(2): 144-152, 2007. PMID: 18158557. DOI: 10.1038/labinvest.3700710
20 Barczak W, Suchorska WM, Sobecka A, Bednarowicz K, Machczynski P, Golusinki P, Rubis B, Masternak MM and Golusinki W: hTERT C250T promoter mutation and telomere length as a molecular markers of cancer progression in patients with head and neck cancer. Mol Med Rep 16(1): 441-446, 2017. PMID: 28535013. DOI: 10.3892/mmr.2017.6590

21 Seow WJ, Cawthon, RM, Purdue, MP, Hu W, Gao Y, Huang W, Weinstein S, Ji B, Virtamo J, Hosgood H, Bassig B, Shu X, Cai Q, Xiang Y, Min S, Chow W, Berndt S, Kim C, Lim U, Albanes D, Caporaso N, Chanock S, Zheng W, Rothman N and Lan Q: Telomere length in white blood cell DNA and lung cancer: A pooled analysis of three prospective cohorts. Cancer Res 74(15): 4090-4098, 2014. PMID: 24853549. DOI: 10.1158/0008-5472.can-14-0459

22 Ohali A, Avigad S, Ash S, Goshen Y, Luria D, Feinmesser M, Zaizov R and Yaniv I: Telomere length is a prognostic factor in neuroblastoma. Cancer 107(6): 1391-1399, 2006. PMID: 16917952. DOI: $10.1002 / \mathrm{cncr} .22132$

23 Wu X, Amos CI, Zhu Y, Zhao H, Grossman BH, Shay JW, Luo S, Hong WK and Spitz MR: Telomere dysfunction: A potential cancer predisposition factor. J Natl Cancer Inst 95(16): 12111218, 2003. PMID: 12928346. DOI: 10.1093/jnci/djg011

24 Wentzensen IM, Mirabello L, Pfeiffer RM and Savage SA: The association of telomere length and cancer: a meta-analysis. Cancer Epidemiol Biomarkers Prev 20(6): 1238-1250, 2011. PMID: 21467229. DOI: 10.1158/1055-9965.epi-11-0005

25 Weischer M, Nordestgaard BG, Cawthon RM, Freiberg JJ, Tybjærg-Hansen A and Bojesen SE: Short telomere length, cancer survival, and cancer risk in 47102 individuals. J Natl Cancer Inst 105(7): 459-468, 2013. PMID: 23468462. DOI: 10.1093/jnci/djt016

26 Gu J, Chen M, Shete S, Amos CI, Kamat A, Ye Y, Lin J, Dinney $\mathrm{CP}$ and $\mathrm{Wu} \mathrm{X}$ : A genome-wide association study identifies a locus on chromosome $14 \mathrm{q} 21$ as a predictor of leukocyte telomere length and as a marker of susceptibility for bladder cancer. Cancer Prev Res 4(4): 514-521, 2011. PMID: 21460395. DOI: 10.1158/1940-6207.capr-11-0063

27 Xie H, Wu X, Wang S, Chang D, Pollock RE, Lev D and Gu J: Long telomeres in peripheral blood leukocytes are associated with an increased risk of soft tissue sarcoma. Cancer 119(10): 1885-1891, 2013. PMID: 23408253. DOI: 10.1002/cncr.27984

28 Bao Y, Prescott J, Yuan C, Zhang M, Kraft P, Babic A, MoralesOyarvide V, Qian Z, Buring J, Cochrane B, Gaziano J, Giovannucci E, Manson J, Ng K, Ogino S, Rohan T, Sesso H, Stampfer M, Fuchs C, De Vivo I, Amundadottir L and Wolpin B: Leucocyte telomere length, genetic variants at the TERT gene region and risk of pancreatic cancer. Gut 66(6): 1116-1122, 2016. PMID: 27797938. DOI: 10.1136/gutjnl-2016-312510

29 Snetselaar R, Oosterhout MFMV, Grutters JC and Moorsel CHMV: telomerase reverse transcriptase polymorphism rs2736100: A balancing act between cancer and non-cancer disease, a meta-analysis. Front Med 5, 2018. PMID: 29536006. DOI: 10.3389/fmed.2018.00041

30 Pang T, Zhou M, Liu R, Luo J and Xia R: TERT rs2736098 (Ex2-659G $>A$ ) polymorphism and cancer susceptibility: evidence from a comprehensive meta-analysis. Oncotarget $8(56)$, 2017. PMID: 29221218. DOI: 10.18632/oncotarget.21703

31 Liu Z, Li G, Wei S, Niu J, Wang LE, Sturgis EM and Wei Q: Genetic variations in TERT-CLPTM1L genes and risk of squamous cell carcinoma of the head and neck. Carcinogenesis 31(11): 19771981, 2010. PMID: 20802237. DOI: 10.1093/carcin/bgq179 
32 Hosgood HD, Cawthon R, He X, Chanock S and Lan Q: Genetic variation in telomere maintenance genes, telomere length, and lung cancer susceptibility. Lung Cancer 66(2): 157-161, 2009. PMID: 19285750. DOI: 10.1016/j.lungcan.2009.02.005

33 Varadi V, Brendle A, Brandt A, Johansson R, Enquist K, Henriksson R, Svenson U, Tavelin B, Roos G, Hemminki K, Lenner P and Försti A: Polymorphisms in telomere-associated genes, breast cancer susceptibility and prognosis. Eur J Cancer 45(17): 3008-3016, 2009. PMID: 19766477. DOI: 10.1016/ j.ejca.2009.08.012

34 Bojesen SE, Pooley KA, Bojesen SE, Pooley KA, Johnatty SE, Beesley J, Michailidou K et al.: Multiple independent variants at the TERT locus are associated with telomere length and risks of breast and ovarian cancer. Nat Genet 45(4): 371-384, 2013. PMID: 23535731. DOI: 10.1038/ng.2566
35 Wang Z, Zhu B, Zhang M, Parikh H, Jia J, Chung CC, Sampson $\mathrm{JN}$ et al.: Imputation and subset-based association analysis across different cancer types identifies multiple independent risk loci in the TERT-CLPTM1L region on chromosome 5p15.33. Human Mol Genet 23(24): 6616-6633, 2014. PMID: 25027329. DOI: $10.1093 / \mathrm{hmg} / \mathrm{ddu} 363$

36 Wang Y, Jiang W, Liu X and Zhang Y: Tankyrase 2 (TNKS2) polymorphism associated with risk in developing non-small cell lung cancer in a Chinese population. Pathol Res Pract 211(10): 766-771, 2015. PMID: 26293798. DOI: 10.1016/j.prp. 2015.07 .003

Received February 15, 2020

Revised March 29, 2020

Accepted March 31, 2020 